\title{
GONTINUOUSLY INFINITE MATRICES AND THE ALGEBRAIC THEORY OF FREDHOLM'S EQUATION
}

\author{
W. B. SMITH-WHITE
}

(received 26 May 1959)

\section{Introduction}

In this note the theory of the integral equation

$$
\phi(x)=f(x)+\int_{a}^{b} K(x, y) \phi(y) d y
$$

is reduced to elementary matrix algebra. The Fredholm theorems are proved without the introduction of the usual parameter $\lambda$ and without using the properties of the resolvent considered as an analytic function of the complex variable $\lambda$. There is an exact correspondence between the properties of the equation (1) and the properties of a finite linear system. Here the proofs of these properties are also in exact correspondence with the same for the elementary case. Only the most fundamental analytic properties of the integral are used and the discussion is the same whether $K(x, y)$ is continuous or in the Lebesgue class $L^{2}$. Though the Fredholm theory has been recounted many times before, the essential simplicity of the present note seems to have been ovetlooked.

\section{Infinite Matrices}

The equation (1) is commonly written in symbolic notation

$$
\phi-K \phi=f
$$

where $\phi, f$ are 'function-vectors' and $K$ is an 'operator'. Here we shall more definitely regard the symbols as matrices and we gain a little by the use of a few obvious properties of such matrices. A very brief description of the notion will suffice.

Take axes in the plane with $O X$ "vertically downwards" and $O Y$ to the right. The region $a \leqq x \leqq b, a \leqq y \leqq b$ corresponds to a square in the plane and the function values $K(x, y)$ attached to the points of this square constitute a matrix $K$ of 'type' $(\infty \times \infty)$, i.e. with infinitely many rows and columns. 
The coordinate $x$ fixes a row of $K$ and the coordinate $y$ fixes a column of $K$. The analogy with a finite array $a_{i j}$ will be apparent.

Matrices of type $(\infty \times m)$ and $(m \times \infty)$ will be used also. The former of these, for example, consists of $m$ functions $p_{1}(x), \cdots, p_{m}(x)$, 'spread' over $m$ parallel lines running from $x=a$ to $x=b$. Such a matrix $P$ would be indicated by the scheme

$$
P=\left(p_{1}(x), \cdots, p_{m}(x)\right)=\left(p_{1}, \cdots, p_{m}\right) .
$$

Here $p_{1}$ is a single column or $(\infty \times 1)$ matrix.

The transposed matrix is defined in the obvious way. The transpose of $K$ will be denoted by $K^{*}$. The transpose of $P$ is

$$
P^{*}=\left(\begin{array}{c}
p_{1}^{*} \\
\vdots \\
p_{m}^{*}
\end{array}\right)
$$

of type $(m \times \infty)$.

Addition and multiplication are defined as for finite matrices. For multiplication the row $\times$ column rule is used and integration replaces summation. In particular $K^{2}$ is the matrix corresponding to the iterated function

$$
K^{(2)}(x, y)=\int_{a}^{b} K(x, s) K(s, y) d s .
$$

If $P, Q$ be the two matrices

$$
P=\left(p_{1} \cdots p_{m}\right), \quad Q=\left(q_{1} \cdots q_{m}\right)
$$

then $P Q^{*}$ is the $(\infty \times \infty)$ matrix corresponding to the function

$$
p_{1}(x) q_{1}(y)+\cdots+p_{m}(x) q_{m}(y)
$$

while $Q^{*} P$ is the $m \times m$ matrix with elements

$$
\int_{a}^{b} q_{i}(s) p_{j}(s) d s .
$$

It will be clear that the usual rules of matrix algebra apply to these infinite matrices. By introducing single columns $f, \phi$ to represent the functions $f, \phi$ the equation (1) may be written in the form (2). Or, if $K$ be a finite matrix $(m \times m)$ and $f, \phi$ be $m$-columns, then (2) represents a finite linear system. This symbolic identity of the finite and infinite cases represents a real similarity in their properties.

The rank of an infinite matrix will be defined as follows. Draw $m$ parallels to each of the axes across the matrix square and number them from 1 to $\mathrm{m}$. Let $K_{i y}$ be the value of $K(x, y)$ at the point of intersection of the $i$-th parallel 
to $O Y$ and the $j$-th parallel to $O X$. The determinant $\left|K_{i j}\right|$ will be called a determinant of order $m$ of the matrix $K$. The rank of $K$ is $m$ if some determinant of order $m$ does not vanish while all determinants of order $m+1$ do vanish. If no value of $m$ satisfies this description then $K$ has rank $\infty$. Corresponding definitions apply to matrices of type $(\infty \times m)$ or $(m \times \infty)$.

The following results are so simple that they may be stated here without proof. Not all of them are used in the sequel.

(i) The $(\infty \times m)$ matrix $P$ has rank $m$ if and only if the functions $p_{1}(x), \cdots, p_{m}(x)$ are linearly independẹnt.

(ii) If $K$ is $(\infty \times \infty)$ and rank $m$ then $K=P Q^{*}$ where $P, Q$ are $(\infty \times m)$ and rank $P=\operatorname{rank} Q=m$.

(iii) If $K, L$ have finite rank so does $K+L$.

(iv) If $P, Q$ are $(\infty \times m)$ and $\operatorname{rank} P=m$ then rank $P Q^{*}=\operatorname{rank} Q^{*}$. In particular if $P Q^{*}=0$ then $Q^{*}=0$, so that a common factor $P$ may be cancelled from a matrix equation.

(v) For any $K, L, \operatorname{rank} K L \leqq\left\{\begin{array}{l}\operatorname{rank} K \\ \operatorname{rank} L\end{array}\right.$.

(vi) For a square $K$ the trace of $K$ is defined by

$$
\operatorname{tr} K=\int_{a}^{b} K(s, s) d s .
$$

Then $\operatorname{tr} K L=\operatorname{tr} L K$. Or if $P, Q$ are $(\infty \times m)$ matrices, $\operatorname{tr} P Q^{*}=\operatorname{tr} Q^{*} P$, where the term on the right is the usual trace of the $(m \times m)$ matrix $Q^{*} P$. Of course, in the Lebesgue theory, $\operatorname{tr} K$ may be meaningless. However,

$$
\operatorname{tr} \bar{K}^{*} K=\iint_{a}^{b}|K(x, y)|^{2} d x d y<\infty
$$

where $\bar{K}^{*}$ is the transposed conjugate of $K$.

(vii) Setting

$$
\|K\|=\sqrt{ }\left(\operatorname{tr} \bar{K}^{*} K\right)
$$

then, for any $K, L$

$$
\begin{aligned}
\|K L\| & \leqq\|K\| \cdot\|L\| \\
\|K+L\| & \leqq\|K\|+\|L\| .
\end{aligned}
$$

These well known results depend on Schwarz's inequality.

To illustrate the conciseness of this notation consider a matrix $P$ of type $(\infty \times m)$. Then $\bar{P}^{*} P$ is hermitian and positive definite of type $(m \times m)$ so there is a square $c$, type $(m \times m)$, such that

$$
\bar{c}^{*} \bar{P}^{*} P c=I
$$

where $I$ is the unit $(m \times m)$. Thus $Q=P c$ is unitary. This means that the functions

$$
q_{1}(x), \cdots, q_{m}(x)
$$


form a unitary-orthogonal set and are linear combinations of $p_{1}(x), \cdots, p_{m}(x)$ This is the Gram-Schmidt orthogonalisation result.

\section{Linear Systems}

The most important difference between the finite square matrices and those of infinite type is that the latter do not contain a unit matrix. Then also there is no inverse to a given matrix. It is this fact which accounts for the differences in the properties of finite linear systems and linear integral equations.

When $a$ is non-singular the solution of the finite system $a \phi=f$ is $\phi=a^{-1} f$, and depends on the existence of the inverse. There is another formal solution which we obtain by writing $a=I-k, a^{-1}=I-k_{1}$. The relations $a a^{-1}=a^{-1} a=I$ then give

$$
k+k_{1}=k k_{1}=k_{1} k
$$

and the solution of

$$
\phi-k \phi=f
$$

is

$$
f-k_{1} f=\phi
$$

This solution (5) of (4) may be derived by the use of (3) only without reference to the existence of the unit $I$ and the inverse $a^{-1}$. Thus, assuming (4) we multiply it by $k_{1}$,

$$
k_{1} \phi-k_{1} k \phi=k_{1} f
$$

and, substituting for $k_{1} k$ from (3), using (4) again, this reduces to (5). Provided relations of the type (3) exist, this solution is equally valid for the integral equation (2). This suggests the following definition:

Definition. We say that matrix $K$ of type $(\infty \times \infty)$ is regular if there exists $K_{1}$ such that

$$
K+K_{1}=K K_{1}=K_{1} K \text {. }
$$

We call $K_{1}$ the 'reciprocal' of $K$. The symmetry shows that $K$ is the reciprocal of $K_{1}$.

Reverting, for a moment, to the finite system $a \phi=f$ we note that the general results for it now follow by transformation. The relations

$$
\phi=c \phi^{\prime}, \quad f^{\prime}=b f
$$

with non-singular $b, c$, transform the equation $a \phi=f$ to the equivalent one $A \phi^{\prime}=f^{\prime}$, where $A=b a c$. At the same time the contragredient relations

$$
g^{\prime}=c^{*} g, \quad \psi=b^{*} \psi^{\prime}
$$


transform the transposed system

$$
a^{*} \psi=g
$$

to $A^{*} \psi^{\prime}=g^{\prime}$. Further $\psi^{\prime *} f^{\prime}=\psi^{*} f$ which states the invariance of the scalar product.

The two fundamental theorems are

THEOREM 1. The homogeneous equations

$$
a \phi=0, \quad a^{*} \psi=0
$$

have the same number $r(\geqq 0)$ of independent solutions. For $r=0$ the statement means that the equations have only the trivial solutions $\phi=0, \psi=0$.

THEOREM 2. Let $\phi_{1}, \cdots, \phi_{r} ; \psi_{1}, \cdots, \psi_{r}$ be independent solutions of (7). The non-homogeneous equation

$$
a \phi=f
$$

has a solution if and only if

$$
\psi_{i}^{*} f=0, \quad i=1, \cdots, r .
$$

Then the general solution is

$$
\phi=\phi_{0}+c_{1} \phi_{1}+\cdots+c_{r} \phi_{r}
$$

where $\phi_{0}$ is a particular solution and $c_{1}, \cdots, c_{r}$ are arbitrary constants. In particular for $r=0$ the equation (8) has a unique solution for any $f$.

The proof of these may be obtained by noting that if the theorems hold for $A$ they hold also for $a$. Then by selecting suitable $b, c$ we may suppose $A$ to be in diagonal form for which the theorems are obviously true.

We shall see that quite similar argument will establish the main Fredholm theorems for the integral equation (2).

\section{Regular Matrices, Equivalence and the Transformation of Fredholm Equations}

If $K$ is regular according to the definition (6), then its reciprocal $K_{1}$ is unique. Thus the two relations

$$
K+K_{1}=K K_{1}, \quad K+K_{1}^{\prime}=K_{1}^{\prime} K
$$

give, on postmultiplying the second by $K_{1}$,

$$
K K_{1}+K_{1}^{\prime} K_{1}=K_{1}^{\prime} K K_{1}=K_{1}^{\prime}\left(K+K_{1}\right)
$$

which reduces to $K K_{1}=K_{1}^{\prime} K$, and to $K+K_{1}=K+K_{1}^{\prime}$ or $K_{1}^{\prime}=K_{1}$.

For regular $K$ the Fredholm equation

$$
\phi-K \phi=f
$$


has the unique solution

$$
\phi=f-K_{1} f .
$$

The argument has already been given. More generally, by the same argurnent, either of the relations

$$
M=L-K L, \quad M-K_{1} M=L
$$

implies the other.

We define a composition or dot product of matrices $K, L$ by the formula

$$
K \cdot L=K+L-K L \text {. }
$$

Then $(K \cdot L) \cdot M=K \cdot(L \cdot M)$. The matrices which are regular according to the definition (6) form a group with this law of composition, the identical element being the matrix 0 , and the group inverse of $K$ being its reciprocal $K_{1}$.

Two matrices $K, K^{\prime}$ are called equivalent if there are regular matrices $L, M$ such that

$$
K^{\prime}=L \cdot K \cdot M \text {. }
$$

If $K$ is regular so is $K^{\prime}$; any two regular matrices are equivalent. The relation of equivalence is reflexive, symmetric and transitive. We may indicate this equivalence by writing $K^{\prime} \sim K$.

If $M$ is regular the correspondence $\phi^{\prime} \rightarrow \phi=\phi^{\prime}-M \phi^{\prime}$ is one-one with the inverse relation $\phi^{\prime}=\phi-M_{1} \phi$. We use such correspondences to transform the Fredholm equation (2) to an equivalent equation.

The relations

$$
\phi=\phi^{\prime}-M \phi^{\prime}, \quad f^{\prime}=f-L f
$$

with regular $L, M$ transform (2) to the equivalent equation $\phi^{\prime}-K^{\prime} \phi^{\prime}=t^{\prime}$, where $K^{\prime}=L \cdot K \cdot M$. At the same time the contragredient relations

$$
g^{\prime}=g-M^{*} g, \quad \psi=\psi^{\prime}-L^{*} \psi^{\prime}
$$

transform the transposed equation $\psi-K^{*} \psi=g$ to the equivalent equation $\psi^{\prime}-K^{\prime *} \varphi=g^{\prime}$.

Also

$$
\psi^{\prime *} f^{\prime}=\psi^{\prime *}(f-L f)=\left(\psi^{\prime *}-\psi^{*} L\right) f=\psi^{*} f
$$

These properties obviously correspond to those quoted in the previous paragraph for finite systems. We may therefore state the following Fredholm theorems for the equation (2) and observe that if they are true for any $K$ they will be true also for any $K^{\prime} \sim K$.

THEOREM 3. The homogeneous equations

$$
\phi-K \phi=0, \quad \psi-K^{*} \psi=0
$$

have the same finite number $r(\geqq 0)$ of independent solutions. For $r=0$ the 
statement means that the equations have only the trivial solutions $\phi=0$, $\boldsymbol{\psi}=\mathbf{0}$.

THEOREM 4. Let $\phi_{1}, \cdots, \phi_{r} ; \varphi_{1}, \cdots, \psi_{r}$ be independent solutions of (9). The non-homogeneous equation

$$
\phi-K \phi=f
$$

has a solution if and only if

$$
\psi_{i}^{*} f=0, \quad i=1, \cdots, r .
$$

Then the general solution is

$$
\phi=\phi_{0}+c_{1} \phi_{1}+\cdots+c_{r} \phi_{r},
$$

where $\phi_{0}$ is a particular solution and $c_{1}, \cdots, c_{r}$ are arbitrary constants. In particular the equation (10) has a unique solution for any $f$.

\section{Proofs of the Theorems}

The proofs are obtained now in two steps. Firstly we show that if $K$ has finite rank then theorems 3 and 4 reduce to theorems 1 and 2 . Secondly we show that for any $K$ there is an S of finite rank with $K \sim S$. It is only in this final step that we use any analysis, apart from what is involved in the definition of an integral.

FIRST STEP. $K$ is of finite rank $m$ say. We write $K=P Q^{*}$. The relations

$$
\begin{gathered}
\xi=Q^{*} \phi \\
\phi=P \xi+f
\end{gathered}
$$

set up a one-one correspondence between the solutions of the Fredholm equation

$$
\phi-P Q^{*} \phi=f
$$

and the solutions of the finite system

$$
\xi-Q^{*} P \xi=Q^{*} f
$$

Thus from (11) and (13), (12) follows immediately and (14) follows if we pre-multiply (13) by $Q^{*}$. Similarly (12) and (14) yield (11) and (13).

In the same way the relations

$$
\eta=P^{*} \psi, \quad \psi=Q \eta
$$

set up a one-one correspondence between the solutions of the homogeneous equations

Further

$$
\psi-Q P^{*} \psi=0, \quad \eta-P^{*} Q \eta=0 .
$$

$$
\varphi^{*} f=\eta^{*} Q^{*} f=\eta^{*}\left(Q^{*} f\right)
$$


It follows that theorems 3 and 4 for $K=P Q^{*}$ reduce to theorems 1 and 2 for $a=I-Q^{*} P$.

SEcond SteP. This depends on two lemmas.

Lemma 1. If $\|K\|<1$ then $K$ is regular.

Set

$$
K_{1}=-K-K^{2}-K^{3}-\cdots .
$$

The series on the right is the Liouville-Neumann series. Its convergence (or convergence in mean square) under the condition $\|K\|<1$ was proved by E. Schmidt (1908). Then clearly

$$
K+K_{1}=K K_{1}=K_{1} K \text {. }
$$

So that $K$ is regular.

LEMma 2. There is an $S$ of finite rank such that $K \sim S$.

From the theory of the integral we may find a polynomial $L(x, y)$ such that

$$
\iint_{a}^{b}|K(x y)-L(x y)|^{2} d x d y<1
$$

If $K, L$ be the corresponding matrices we set $K=L+M$ then $L$ has finite rank and $\|M\|<1$, so $M$ is regular. If $S=L-M_{1} L$ then $S$ has finite rank and $L=S-M S$. Thus

$$
K=M+L=M+S-M S=M \cdot S \sim S .
$$

\section{Conclusion}

We need the following lemma to complete the account.

Lemma. Suppose $K=P Q^{*}$ is of rank $m$. Then $K$ is regular if and only if $k=Q^{*} P$ is regular.

(i) Suppose $K$ is regular. Then

$$
K_{1}=K K_{1}-K=P Q^{*} K_{1}-P Q^{*}=P R^{*}
$$

say. Hence

$$
P Q^{*}+P R^{*}=P Q^{*} P R^{*}
$$

From this relation we may cancel $P$ in front and then postmultiply by $P$ to obtain

$$
k+k_{1}=k k_{1}
$$

where $k_{1}=R^{*} P$. This means that $k$ is regular or equivalently the determinant $|I-k| \neq 0$.

(ii) Suppose $k$ is regular. If $a=I-k,|a| \neq 0$ and

$$
I-a^{-1}=-k a^{-1}=-a^{-1} k \text {. }
$$

Multiply in front by $P$ and behind by $Q^{*}$. We get 


$$
K+K_{1}=K K_{1}=K_{1} K
$$

where $K_{1}=-P a^{-1} Q^{*}$. Hence $K$ is regular.

If $K(x, y)$ is continuous we say that $K$ is continuous. We may show then that $K_{1}$ is also continuous. This is obvious from the formula $K_{1}=-\mathrm{Pa}^{-1} Q^{*}$ when $K$ has finite rank. In general it follows by the argument in the second step of $\S 5$ since then $L, M$ and so $S$ are continuous.

If $K$ is not regular then $\phi=K \phi$ has a non-trivial solution. This is obvious from the lemma and the correspondence of the first step of $\S 5$ when $K$ has finite rank. In general it follows as above.

We define a regular $K$ by the two conditions (6). But one only of these relations is sufficient. For if, for instance,

$$
K+K_{1}=K_{1} K
$$

and $K$ were not regular, we would have a non-zero $\phi$ with $K \phi=\phi$. This gives successively

$$
\begin{aligned}
K_{1} K \phi & =K_{1} \phi \\
K \phi+K_{1} \phi & =K_{1} \phi, \\
K \phi & =0, \\
\phi & =0
\end{aligned}
$$

a contradiction.

University of Sydney. 